\title{
Influência de fatores ambientais, cor e altura de armadilhas na captura do caruncho do bambu
}

\author{
Edilson Soares Silveira ${ }^{1}$, Rudieli Machado Silva ${ }^{2}$, Antonia Railda Roel ${ }^{2}$, Marney Pascoli \\ Cereda $^{2}$ \\ ${ }^{1}$ Instituto Federal de Mato Grosso do Sul - IFMS, Campi Campo Grande, Campo Grande, Mato Grosso do Sul, Brasil. E-mail: \\ edilson.silveira@ifms.edu.br \\ ${ }^{2}$ Universidade Católica Dom Bosco, Campo Grande, Mato Grosso do Sul, Brasil. E-mail: rudiely_mds@ @otmail.com, \\ edilsonbio@hotmail.com, cereda@ucdb.br
}

Recebido: 18/05/2017; Aceito: 04/09/2017

\section{RESUMO}

O objetivo deste trabalho foi avaliar o efeito da influência dos fatores ambientais, cor e altura de instalação de armadilhas com toletes de bambu na captura do Dinoderus minutus, considerado a principal praga pós-colheita do bambu. A literatura aponta a cor e altura das armadilhas como elementos capazes de influenciar na captura deste inseto. Para avaliar essas hipóteses, foram fabricadas 30 armadilhas de garrafas PET nas cores amarela, preta, branca, azul e transparente. Toletes de bambu Bambusa vulgaris foram cortados e secos em uma estufa a $50 \pm 3^{\circ} \mathrm{C}$ durante $48 \mathrm{~h}$. Em cada armadilha foi inserido um tolete de bambu na posição vertical. As armadilhas foram instaladas a 1,5 m e 2,5 m de altura em relação ao solo, em uma touceira de $B$. vulgaris. No período do experimento foram capturados 3301 carunchos. Houve uma correlação positiva entre a captura de carunchos e a pluviosidade, porém não houve correção entre a captura de carunchos e a temperatura. A altura influenciou na captura de D. minutus de modo que um maior número de insetos foi encontrado nas armadilhas instaladas a $2,5 \mathrm{~m}$ do solo. Não foi detectada influência da cor das armadilhas na captura de carunchos, tampouco da interação entre cor e altura de instalação das mesmas.

Palavras-chave: Bambusa, cor, Dinoderus minutus

\section{Influence of environmental factors, color and height of installation of traps with bamboo tops in the capture of the bamboo borer}

\begin{abstract}
The objective of this study was to evaluate the influence of environmental factors, color and height traps facility with bamboo stalks in capturing Dinoderus minutus, considered the main pest postharvest bamboo. The literature shows the color and height as elements able to influence the capture of this insect. To evaluate these hypotheses were made 30 pitfalls of PET bottles in the colors yellow, black, white, blue and transparent. Bambusa vulgaris bamboo stalks were cut and dried in an oven at $50 \pm 3^{\circ} \mathrm{C}$ for $48 \mathrm{~h}$. In each trap was inserted bamboo tolete upright. The traps were placed at $1.5 \mathrm{~m}$ and $2.5 \mathrm{~m}$ high from the ground in a clump of $B$. vulgaris. In the experimental period they were captured borers 3301. There was a positive correlation between the catching borers and rainfall, but this was not found for the temperature. The height influences the capture $D$. minutus so that a greater number of insects found in traps installed $2.5 \mathrm{~m}$ above the ground. It was detected influence of the color of the traps in catching borers, nor the interaction between color and height of installation of the same.
\end{abstract}

Key words: Bambusa, color, Dinoderus minutus 


\section{Introdução}

Dependendo das condições de armazenamento, o bambu pode ser atacado pelo caruncho Dinoderus minutus, Fabricius 1775 (Bostrichidae), podendo reduzir os colmos a pó em poucos meses. Conforme Nair e Mathew (1984) e Borgemeister et. al. (1999), o D. minutus é amplamente distribuído nos trópicos. O principal problema deste caruncho é seu potencial de ataque em relação à alguns produtos de subsistência como, batata, cereais, mandioca, milho, inhame e principalmente o bambu pós-colheita sendo, por este motivo, considerada a principal praga do bambu (PLANK, 1948). Ainda segundo o autor, tanto as larvas como os adultos penetram em bambus armazenados usando feridas ou as extremidades cortadas, realizando túneis perpendiculares às fibras parenquimáticas em torno dos colmos, onde os ovos são depositados.

Este inseto foi descrito cientificamente pela primeira vez em 1775, pelo entomologista dinamarquês Johan Christian Fabricius (1745-1808). Pertencente à família Bostrichidae que apresenta em torno de 520 espécies descritas, entre as quais mais de 100 espécies são encontradas entre o sul da América do Norte e a América do Sul (OLIVEIRA et al., 1986), este inseto é cosmopolita, segundo Spilman (1982), adaptando-se muito facilmente em diferentes regiões do mundo, sendo comum em estoques de bambu e alimentos secos. Diferencia-se dos demais insetos bostrichideos pelas duas suaves depressões arredondadas no dorso do pronoto, bem como nas extremidades próximas aos élitros (LOPEZ, 1974). O tamanho do adulto varia de 3 a $4 \mathrm{~mm}$ de comprimento e largura de 1 a $1,5 \mathrm{~mm}$, cor castanho-avermelhada e preto-acastanhada e corpo cilíndrico (GALLO et al., 2003).

Para Calegari et al. (2007), além de ser considerado um excelente sequestrador de carbono, o interesse pela utilização do bambu tem aumentado em nível mundial por ser um recurso perene, renovável, de rápido crescimento, grande produtividade por área, baixo custo e diversidade na utilização. Em áreas de armazenamento de varas cortadas de bambu, o D. minutus pode tornarse numeroso a ponto de causar sérios danos, reduzindo o bambu a pó ou fibras (SINGH; BHANDARI, 1988).

Azzini et al. (1998) indicam ser o elevado teor de amido dos colmos maduros do bambu, um dos atrativos para o ataque do caruncho. Essa influência seria explicada por Flechtmann et al. (1997a), quando os insetos Bostrichideos, atraídos por fatores químicos e/ou físicos estão próximos de seus hospedeiros. Plank e Hangeman (1951) também afirma existir uma relação entre o teor de amido encontrado no bambu e o ataque do D. minutus. Essa atração explicaria ataques de intensidades diferentes às partes do colmo.

Segundo Liese (2004), o nó do Bambusa vulgaris é a parte do colmo com maior durabilidade em comparação com as partes central e do ápice. Para o autor, a explicação estaria no fato de que a região interna do colmo contém maior concentração de amido armazenado que a região externa. Azzini et al. (1987), também citam a predominância de tecido parenquimático rico em amido na região interna do colmo do B. vulgaris.

Norhisham et al. (2013) afirmam que as mudanças climáticas devem ser consideradas para a captura destes carunchos, visto que o clima influencia o desenvolvimento dos insetos; há relatos de efeito da umidade relativa do ar (UR) na eclosão dos ovos. Os autores relatam que os ovos se desidratam quando em baixa UR, o que ocasiona o encolhimento tanto do embrião como do córion, impedindo a liberação larval. Por outro lado, a UR próxima a $85 \%$ resulta em mortalidade dos ovos. Para Wellians e Singh (1951), além da UR, o fotoperíodo também exerce influência sobre o comportamento do D. minutus, Plank (1948) observa que o D. minutus é mais ativo em baixa luminosidade.

Os olhos compostos desses insetos podem perceber diferentes intensidades de cores, desde que se situem dentro do seu campo de visão monocromática. (MARANHÃO, 1976). Fraga et al. (2011) relatam que os insetos são atraídos ou repelidos por comprimentos de ondas luminosas, contudo, há contradições na literatura com relação à radiação luminosa que mais atrai os insetos. Segundo Kirk (1986), os insetos voadores são capturados com maior facilidade por armadilhas de uma cor específica, enquanto outros podem ser capturados por armadilhas de várias cores. Niemeyer (1985) explica que quando a cor se destaca em relação ao meio em que se encontra exercerá grande influência na captura de alguns insetos.

Para Carvalho (1986) os insetos são atraídos por radiações luminosas distintas da visão humana; Flechtmann et al. (1997a) relatam que as cores preta, marrom e verde exercem influência mínima na atratividade de insetos bostrichideos, enquanto a ausência de cor (transparência) e as cores branca e amarela, geralmente encontradas em plantas hospedeiras, atuam como atraentes. Hosking (1979) demonstrou que o uso de armadilhas para a captura de insetos voadores é essencial no estudo dos insetos. A altura, até certo ponto, é também um fator físico que pode influenciar na captura de insetos. Flechtamann et al. (1997b) mostraram que existe uma tendência de insetos Bostrichideos voarem preferencialmente entre 1,0 m e 2,0 m. Peres Filho et al. (2012) concluíram que quanto maior a altura de instalação de armadilhas, maior o número de insetos capturados, o que foi comprovado em armadilhas instaladas em Pinus caribaea $v$. hondurensis, onde na altura de 2,0 $\mathrm{m}$ foi capturado mais insetos. 
Segundo Gomes et al. (1982), o clima da região Centro-Oeste do Brasil apresenta duas estações bem definidas ao longo do ano: inverno e verão. O inverno é seco e o verão quente e úmido. Os sistemas atmosféricos que atuam nesta região são tanto de origem tropical quanto extratropical. O setor mais ao norte sofre influência de sistemas que atuam na Amazônia, já o setor sul (região do estudo) sofre influência de sistemas extratropicais tais como, frentes frias e linhas de instabilidade (REBOITA et al., 2010).

O objetivo deste trabalho foi avaliar o efeito da influência da altura, cor de armadilhas com toletes de bambus e pluviosidade na captura do D. minutus, como alternativa de controle para coleta massal deste inseto

\section{Material e Métodos}

$\mathrm{O}$ experimento foi realizado de 25 de novembro a 20 de dezembro de 2012, na Fazenda Escola, Base de Pesquisa em Ciências Agrárias da Universidade Católica Dom Bosco, no Centro de Tecnologia e Análise do Agronegócio (CeTeAgro), município de Campo Grande - MS. Neste período, o clima correspondeu ao verão quente e úmido.

O clima da região de Campo Grande, segundo a classificação de Kopen, pode ser considerado como de transição entre clima subtropical úmido (Cfa), clima Tropical Úmido (Aw) e de Savana (REBOITA et al., 2010).

A espécie B. vulgaris foi selecionada por ser citada na literatura como uma das mais atacadas pelo caruncho (OLIVEIRA et al., 2002). Foram coletadas varas maduras de $B$. vulgaris de uma touceira, com pelo menos 20 anos de plantio, com coordenadas geográficas 20²3'14' ' S e 5436'29' ' W, e 532 metros de altitude. As varas foram cortadas em 150 toletes $(25 \mathrm{~cm}$ de altura x $4 \mathrm{~cm}$ x $1 \mathrm{~cm}$ ). Em seguida, os toletes foram colocados em uma estufa com renovação e circulação de ar, ajustada a $50 \pm 3^{\circ} \mathrm{C}$ por 48 horas.

Foram fabricadas 30 armadilhas com toletes de bambu, utilizando garrafas transparentes de polietileno treftalato (PET), que foram cortadas transversalmente $20 \mathrm{~cm}$ acima da base, descartando-se a parte superior. Em seguida cada armadilha foi totalmente revestida externamente, com adesivo nas cores amarela, branca, azul, preta ou transparente, realizando-se três perfurações de $12 \mathrm{~mm}$ na base de cada uma delas para evitar acúmulo de água, em caso de chuvas.

Estas armadilhas foram instaladas na mesma touceira de onde foram retiradas os toletes de bambu, em posição vertical e sentido norte-sul. As alturas selecionadas foram de $1,5 \mathrm{~m}$ e $2,5 \mathrm{~m}$ em relação ao solo (Figura 1), por incluir o intervalo citado por Flechtmaann et al. (1997b), ou seja, entre 1,0 m e 2,0 m, com $0,5 \mathrm{~m}$ de amplitude. Em cada altura foram instaladas 15 armadilhas coloridas, distanciadas $40 \mathrm{~cm}$ entre si. Cada armadilha colorida foi repetida três vezes em cada altura e a ordem das mesmas, foi obtida por sorteio. Em seguida foi introduzido em cada armadilha, um tolete de B. vulgaris desidratado na posição vertical.

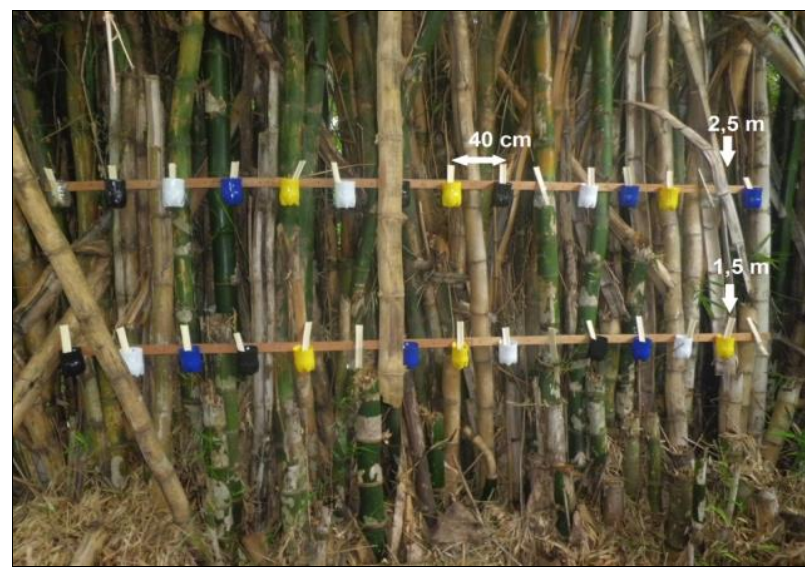

Figura 1 - Distribuição das armadilhas coloridas com toletes de bambu em duas alturas (1,5 e 2,5m) no sentido norte-sul da touceira de Bambusa vulgaris para avaliar a captura de Dinoderus minutus em campo, de 25 de novembro a 20 de dezembro de 2012, Campo Grande, MS.

Foram realizadas cinco coletas de carunchos, com intervalos de cinco dias entre elas. No intervalo entre as coletas, as armadilhas eram inspecionadas para limpeza e manutenção. Para cada experimento, foram utilizados 30 toletes de bambu, totalizando 150 durante todas coletas. Ao final de cada coleta, os carunchos foram retirados dos toletes no laboratório de entomologia da Universidade Católica Dom Bosco (UCDB), manualmente, com auxílio de uma lupa manual e bisturi. Em seguida os mesmos foram inseridos em recipientes de vidro de $100 \mathrm{~mL}$. A temperatura máxima e mínima, bem como a pluviosidade média foram obtidos de uma estação meteorológica local, modelo Vantage Pro2.

O delineamento estatístico utilizado foi inteiramente casualizado em esquema fatorial 5 x 2 (cinco tratamentos (cores) e duas alturas) com três repetições de cada cor por altura.

Os dados foram analisados pela análise da variância paramétrica (ANOVA), utilizando o software The SAS System 9.0, a fim de avaliar o efeito da cor, da altura e da interação entre cor e altura. Uma vez desenvolvido o modelo de ANOVA, foi avaliada a distribuição da normalidade dos resíduos através do teste Shapiro-Wilk, com o objetivo de verificar se os dados atendem as premissas da análise paramétrica. Também foi utilizado o Coeficiente de Correlação de Pearson para avaliar a correlação da temperatura e a pluviosidade com a quantidade de carunchos capturados. 


\section{Resultados e Discussão}

\section{Influência da pluviosidade}

Em relação às condições climáticas na época do experimento, a temperatura mínima variou de $23,4{ }^{\circ} \mathrm{C}$ a $19,2{ }^{\circ} \mathrm{C}$, enquanto a temperatura máxima variou de 32,9 ${ }^{\circ} \mathrm{C}$ a $22,3{ }^{\circ} \mathrm{C}$ e a pluviosidade, variou de 0 a $49,0 \mathrm{~mm}$. Essas condições climáticas correspondem ao verão quente e úmido, típicas das regiões de Cerrado da região Centro-Oeste que, segundo Plank (1948), favorecem o aumento populacional de carunchos.

Dentre as cinco coletas realizadas, a segunda foi a que apresentou maior captura de carunchos, com 1783 indivíduos, correspondendo aos dias com maiores temperaturas e índices pluviométricos. A relação entre a quantidade de carunchos capturados e as condições ambientais favoráveis foi também relatada por Bousquet (1990), com maior ocorrência natural de D. minutus em coletas realizadas em regiões com temperatura e pluviosidades mais elevadas. As duas últimas coletas apresentaram menor número de carunchos capturados, coincidindo com a ausência de pluviosidade no período. Considerando que ocorreu um pico de quantidade total de carunchos capturados, na segunda coleta, o período sobressaiu bastante em relação às demais coletas, onde a quantidade total de captura foi relativamente homogênea (Tabela 1), sendo este período o melhor para a análise dos dados.

Para confirmar a hipótese de que temperatura e umidade elevadas favorecem a captura de carunchos, foi estabelecida a correlação simples (Tabela 2). Esta análise indicou que houve uma correlação positiva $(r=0,60)$ entre a captura de carunchos e a pluviosidade, com resultados estatisticamente significativos, porém o mesmo não foi encontrado para a temperatura.

Tabela 2. Correlações entre a quantidade de carunchos capturados e as temperaturas mínima, máxima, média e pluviosidade média, segundo Coeficiente de Correlação de Pearson, de 25 de novembro a 20 de dezembro de 2012, Campo Grande, MS.

\begin{tabular}{ccc}
\hline Variável & Média & Correlação (r) \\
\hline Temperatura mínima & 21,72 & 0,09 \\
Temperatura máxima & 30,58 & 0,18 \\
Temperatura média & 26,12 & 0,16 \\
Pluviosidade média & 10,84 & 0,60 \\
\hline
\end{tabular}

Tabela 1. Variação das temperaturas mínima, máxima e média $\left({ }^{\circ} \mathrm{C}\right)$, pluviosidade diária $(\mathrm{mm})$ e número de carunchos Dinoderus minutus capturados, período de 25 de novembro a 20 de dezembro de 2012, Campo Grande, MS.

\begin{tabular}{|c|c|c|c|c|c|}
\hline \multirow[b]{2}{*}{ Data } & \multicolumn{3}{|c|}{ Temperatura $\left({ }^{\circ} \mathrm{C}\right)$} & \multirow{2}{*}{$\begin{array}{l}\text { Pluviosidade diária } \\
\qquad(\mathrm{mm})\end{array}$} & \multirow{2}{*}{$\begin{array}{l}\text { Número de carunchos } \\
\text { capturados }\end{array}$} \\
\hline & Mínima & Máxima & Média & & \\
\hline $25 / 11 / 2012$ & 21,1 & 30,3 & 25,7 & 0,0 & - \\
\hline $26 / 11 / 2012$ & 20,5 & 30,7 & 25,6 & 0,0 & - \\
\hline $27 / 11 / 2012$ & 21,2 & 30,2 & 25,7 & 0,0 & - \\
\hline $28 / 11 / 2012$ & 20,6 & 32,6 & 26,6 & 0,0 & - \\
\hline $29 / 11 / 2012$ & 20,4 & 31,3 & 25,8 & 25,0 & - \\
\hline $30 / 11 / 2012$ & 19,2 & 29,3 & 24,2 & 29,4 & 577 \\
\hline $01 / 12 / 2012$ & 21,2 & 28,8 & 25,0 & 49,0 & - \\
\hline $02 / 12 / 2012$ & 20,6 & 30,4 & 25,5 & 28,0 & - \\
\hline 03/12/2012 & 21,2 & 31,6 & 26,4 & 23,0 & - \\
\hline $04 / 12 / 2012$ & 21,8 & 31,1 & 26,4 & 3,4 & - \\
\hline $05 / 12 / 2012$ & 22,4 & 31,2 & 26,8 & 23,8 & 1783 \\
\hline $06 / 12 / 2012$ & 22,8 & 31,1 & 26,9 & 2,0 & - \\
\hline $07 / 12 / 2012$ & 22,6 & 31,3 & 26,9 & 22,2 & - \\
\hline $08 / 12 / 2012$ & 23,4 & 32,4 & 27,9 & 0,2 & - \\
\hline $09 / 12 / 2012$ & 21,8 & 32,9 & 27,3 & 0,4 & - \\
\hline $10 / 12 / 2012$ & 22,8 & 32,7 & 27,7 & 0,0 & 441 \\
\hline $11 / 12 / 2012$ & 21,7 & 31,5 & 26,6 & 0,0 & - \\
\hline $12 / 12 / 2012$ & 22,7 & 31,3 & 27,0 & 0,0 & - \\
\hline $13 / 12 / 2012$ & 20,4 & 30,2 & 25,3 & 0,0 & - \\
\hline $14 / 12 / 2012$ & 20,5 & 28,2 & 24,3 & 0,0 & - \\
\hline $15 / 12 / 2012$ & 21,2 & 28,1 & 24,6 & 1,0 & 259 \\
\hline $16 / 12 / 2012$ & 21,2 & 27,1 & 24,1 & 0,0 & - \\
\hline $17 / 12 / 2012$ & 20,0 & 22,3 & 21,1 & 0,0 & - \\
\hline $18 / 12 / 2012$ & 19,7 & 29,8 & 24,7 & 0,0 & - \\
\hline $19 / 12 / 2012$ & 21,9 & 30,8 & 26,3 & 0,0 & - \\
\hline $20 / 12 / 2012$ & 23,0 & 31,6 & 27,3 & 0,0 & 241 \\
\hline
\end{tabular}


Influência da altura de instalação das armadilhas com toletes de bambu

Considerando as duas alturas em que foram instaladas as armadilhas, durante todo o experimento foram capturados 3301 carunchos. Observou-se que armadilhas na altura de 1,5 m capturaram em média 53 carunchos de um total de 1337 capturas. Na altura de 2,5 m, ocorreu maior captura com uma média de 78 carunchos de um total de 1964 capturas.

Sendo assim, comparando somente as alturas, independente das cores das armadilhas, os resultados apresentaram diferenças estatísticas a $5 \%$ de probabilidade pela análise de variância (ANOVA), de modo que um maior número de insetos foi encontrado nas armadilhas instaladas a $2,5 \mathrm{~m}$ em relação ao solo (Tabela 3). Estes resultados confirma os reportados por Flechtmann et al. (1997b) e Peres Filho et al. (2012), de que a altura influencia na quantidade de insetos bostrichideos capturados em campo com maior incidência em alturas elevadas. A altura de $2,5 \mathrm{~m}$ é a que mais se aproxima da altura citada por Flechtmann et al. (1997b), que em experimentos com armadilhas coloridas instaladas entre $0,5 \mathrm{~m}$ e $10,0 \mathrm{~m}$ de altura, em Pinus caribaea $v$. hondurensis, capturou maior número de insetos Bostrichidae na altura de 2,0 m.

Um dos motivos de maior captura com armadilhas a 2,5 m de altura se deve, provavelmente, ao encontro dos parceiros sexuais nessa faixa de altura do bambu. Existe registros na literatura de feromônios sexuais e de agregação encontrados frequentemente em pragas de produtos armazenados (NORDLUND; LEWIS, 1976).

Segundo Boughton e Fadamiro (1996), machos da espécie de insetos Bostrichídeos, que dependem principalmente de alimentos para se reproduzirem, produzem feromônios de agregação para atrair indivíduos de ambos os sexos para as fontes de alimentos, propiciando maior sucesso de acasalamento. Nesse sentido, faz-se necessário determinar se há ou não liberação de feromônios de agregação e/ou sexual desta espécie nesta faixa de altura. Caso sejam encontrados, toletes de bambu impregnados com feromônios sexuais naturais e/ou sintéticos podem promover maior captura de carunchos.

Tabela 3. Captura de Dinoderus minutus em armadilhas de toletes de bambu instaladas a $1,5 \mathrm{~m}$ e a $2,5 \mathrm{~m}$ de altura, de 25 de novembro a 20 de dezembro de 2012, Campo Grande, MS

\begin{tabular}{cccc}
\hline \multirow{2}{*}{ Altura } & \multicolumn{3}{c}{ Quantidade de carunchos capturados } \\
\cline { 2 - 4 } & Total & Média & Erro Padrão $( \pm)$ \\
\hline $1,5 \mathrm{~m}$ & 1337 & 53,48 & 1,58 \\
$2,5 \mathrm{~m}$ & 1964 & 78,56 & 2,14 \\
\hline $\mathrm{F}$ & \multicolumn{3}{c}{6,10} \\
$\mathrm{p}$ & $0,0181^{*}$ \\
\hline * Significativo ao nível de $5 \%$ & de probabilidade pela análise de \\
variância (ANOVA). [Transformação utilizada = 1/ln (quantidade de \\
carunchos coletados)].
\end{tabular}

Interação entre as cores das armadilhas feitas com toletes de bambu e a altura

Quanto ao total de carunchos coletados, as amostragens realizadas com as armadilhas de diferentes cores nas duas alturas em relação ao solo não apresentaram diferenças significativas para a influência desta interação. Considerando a média de cinco coletas, observou-se que na altura de $1,5 \mathrm{~m}$ a armadilha de cor branca capturou mais carunchos, com média de 67 indivíduos, seguida pela de cor azul (54), transparente (51), preto (49) e amarelo (45). Na altura de 2,5 m, a armadilha de cor transparente capturou mais carunchos, com média de 89 indivíduos, seguida pela de cor branca (83), preta (77), azul (76) e amarela (65). O p-valor obtido foi de 0,9980, sendo assim, pode-se afirmar estatisticamente, que nas alturas de $1,5 \mathrm{~m}$ e $2,5 \mathrm{~m}$ a quantidade média de carunchos capturados é a mesma independente da cor da armadilha.

Apesar de não apresentar resultados significativos, considerando a média das cinco coletas, as armadilhas de coloração branca foram responsáveis pela captura de 150 carunchos $(23,17 \%$ do total coletado) e, no que se refere à altura das armadilhas, observou-se que 59,45\% dos exemplares capturados correspondem às armadilhas instaladas a 2,5 m de altura em relação ao solo.

\section{Influência das cores das armadilhas com toletes de bambu}

Considerando somente as cores das armadilhas, os resultados também não foram significativos, pois foi observado que a quantidade de carunchos capturados é semelhante em todas as armadilhas coloridas (Tabela 4). Estes resultados diferem dos relatados por Azeredo (2006) em avaliações de capturas de insetos-praga associados à jabuticaba (Myrciaria jabuticaba) com armadilhas coloridas, onde a coloração branca foi uma das mais eficazes na captura. A literatura tem comprovado a influência da cor da armadilha na captura de vários grupos de outros insetos, porém, neste estudo esta influência não pôde ser comprovada, sendo importante destacar que todas as armadilhas coloridas capturaram carunchos.

Tabela 4. Número médio e respectiva significância de carunchos Dinoderus minutus capturados em armadilhas coloridas com toletes de bambu, independente da altura de instalação, no período de 25 de novembro a 20 de dezembro de 2012, Campo Grande, MS.

\begin{tabular}{lcc}
\hline Cor & \multicolumn{2}{c}{ Quantidade de carunchos capturados } \\
\cline { 2 - 3 } & Média de 5 coletas & Erro padrão $( \pm)$ \\
\hline Branca & $75,50^{\mathrm{a}}$ & 2,16 \\
Transparente & $70,70^{\mathrm{a}}$ & 1,72 \\
Preta & $63,30^{\mathrm{a}}$ & 1,52 \\
Azul & $65,20^{\mathrm{a}}$ & 1,52 \\
Amarela & $55,40^{\mathrm{a}}$ & 1,79 \\
\hline \multicolumn{1}{c}{$\mathrm{p}$} & $0,6106^{*}$ & \\
\hline
\end{tabular}

Média seguidas da mesma letra não difere entre si pela ANOVA. 
Em experimento de captura com uso de armadilhas coloridas, Gorski (2003) e Kim et al. (1999), comprovaram que para a mosca branca (Bemisia tabaci) a cor amarela apresentou maior número de insetos capturados. Resultados semelhantes foram observados por Ott et al. (2006), com armadilhas adesivas para captura de cigarrinhas em pomares de laranja. Na captura de D. minutus em armadilhas coloridas, as de cor amarela não diferiram das demais em número de carunchos capturados. Flechtmann et al. (1997b), em experimentos de captura de insetos Bostrichideos com armadilhas coloridas observaram menor captura de insetos com armadilhas de cor amarela.

Em relação à avaliação da distribuição da normalidade dos resíduos através do teste Shapiro-Wilk, considerando os fatores pesquisados (cor, altura, cor $\mathrm{x}$ altura), demonstrou que que as premissas da análise paramétrica foram satisfeitas, garantindo resultados válidos, pois foi encontrado um p-valor igual a 0,4792.

Os resultados obtidos indicam que a utilização de armadilhas com toletes de bambu, independente da cor, pode constituir um método alternativo de coleta de $D$. minutus, quando instaladas na altura de $2,5 \mathrm{~m} \mathrm{em}$ touceiras de bambu $B$. vulgaris, pois o experimento permitiu a captura de grande número de insetos em curto espaço de tempo. Desta forma, é possível a obtenção de maior número de indivíduos para criação massal em laboratório e que podem ser utilizados em pesquisas futuras

\section{Conclusões}

Quanto maior a pluviosidade maior a quantidade de carunchos capturados. Armadilhas com toletes de bambu instaladas na altura de 2,5 m favoreceram maior captura. Apesar de não terem sido observadas diferenças significativas, todas as armadilhas com toletes de bambu capturaram carunchos. $\mathrm{O}$ emprego de armadilhas a 2,5 $\mathrm{m}$ de altura em períodos chuvosos sugeriram maior captura do caruncho do bambu com o objetivo de criação massal em laboratório para eventuais pesquisas futuras.

\section{Referências Bibliográficas}

AZEREDO, E. H. Comparação e eficiência de cores em armadilha modelo extrato-etanóico com aletas na captura de insetos-praga associados à Myrciaria jabuticaba (Berg) (Mirtaceae). Revista Universidade Rural, Rio de Janeiro-RJ, v. 26, n. 2, p. 54-67, 2006.

AZZINI, A.; ARRUDA, M. C. Q.; FILHO, M. T.; SALGADO, A. L. B.; CIARAMELO, D. Variações dos teores de fibras celulósicas e amido no colmo de bambu. Bragantia, Campinas-SP, v. 46, n. 1, p. 141-145, 1987.
AZZINI, A.; GONDIM-TOMAZ, R. M. A.; ERISMANN, N. M. Desfibramento de cavacos laminados de Bambusa vulgaris Schrad, visando à extração de amido. Bragantia, CampinasSP, v. 7, n. 1, p. 41-44, 1998.

BORGEMEISTER, C.; SCHAFER, K.; GOERGEN, G.; AWANDE, S.; SETAMOU, M.; POEHLING, H. M.; SCHOLZ, D. Hostfinding behavior of Dinoderus bifoveolatus (Coleoptera: Bostrichidae), an important pest of stored cassava: the role of plant volatiles and odors of conspecifics. Journal Annals of the Entomological Society of America, New York, v. 92: p. 766-771, 1999.

BOUGHTON, A.; FADAMIRO, H. Y. Effect of age and sex on the response of walking Prostephanus truncatus (Horn) (Coleoptera, Bostrichidae) to its male produce aggregation pheromone. Journal of Stored Products Research. Amsterdam, v. 32, p. 13-20, 1996.

BOUSQUET, Y. Beetles associated with storede products in Canadá: An identification guide. Canadian Cataloguing in Publication. Ottawa, Canadá, v. 1, p. 68, 1990.

CALEGARI, L.; HASELEIN, C. R.; SCARAVELLI, T. L.; SANTINI, E.J.; STANGERLIN, D. M.; GATTO, D. A.; TREVISAN, R. Desempenho físico-mecânico de painéis fabricados com bambu (Bambusa vulgaris Schr.) em combinação com madeira. Cerne, Lavras-MG, v. 13, n. 1, p. 57-63, 2007.

CARVALHO, J. P. Introdução à entomologia agrícola. Fundação Calouste Gulbenkian, Lisboa, p. 361, 1986.

GOMES, I. A.; PALMIERI, F.; FREITAS, F. G.; BARUQUI, A. M.; MOTA, F.; NAIME, U. J.; SANTANA, D. P.; MOTHCI, E. P.; SANTO, H. G. Levantamento de reconhecimento de média intensidade dos solos e avaliação da aptidão agrícola das terras do Triângulo Mineiro. Rio de Janeiro-RJ: Embrapa-SNLCS; 1982. p. 526. (Boletim de Pesquisa, 1)

FRAGA, D. F.; GRIGOLLI, J. F. J.; LOPES, D. O. P.; SOUZA, L. A.; BUSOLI, A. C. Atratividade de Doru luteipes às armadilhas coloridas na cultura do milho. FAZU em Revista, Uberaba-MG, v. 1, n. 8, p. 15-19, 2011.

GORSKI, R. Evaluation of the effectiveness of natural essential oils in the monitoring of the occurrence of greenhouse whitefly (Trialeurodes vaporariorum Westwood). Jornal of Plant Protection, Poland, v. 4, n. 43, p. 393-397, 2003.

FLECHTMANN, C. A. H.; TEIXEIRA, E. P.; GASPARETO, C. L. Influência das cores na atração de Bostrichidae (Coleoptera) em área de pinheiro tropical em Agudos, Revista Instituto Florestal, São Paulo-SP, v. 9, n. 1, p. 1-17, 1997a.

FLECHTMANN, C. A. H.; TEIXEIRA, E. P.; GASPARETO, C. L. Altura de voo de Bostrichidae (coleóptera) em Pinus caribaea $v$. hondurensis em Agudos, SP. Revista Instituto Florestal, São Paulo-SP, v. 9, n. 1, p. 19-26, 1997 b.

GALLO, D; NAKANO, O; SILVEIRA, N. S; CARVALHO, R. P. L; BERTI, F. E; GORSKI, R. Evaluation of the effectiveness of natural essential oils in the monitoring of the occurrence of greenhouse whitefly (Trialeurodes vaporariorum Westwood). Jornal of Plant Protection Research, Poland, v. 45, n. 4, p. 393-397, 2003. 
HOSKING, G. P. Trap comparison in the capture of flying Coleoptera. New Zealand Entomologist, New Zealand, v. 7, n. 1, p. 87-92, 1979.

KIM, J. K.; PARK, J. J.; PARK, C. H.; PARK, H. S.; CHO, K. J. Implementation of yellow sticky trap for management of greenhouse whitefly in cherry tomato greenhouse. Journal of Korean Society Horticultural Science, Annapolis, v. 40, n. 5, p. 549-553, 1999.

KIRK, W. D. J. Ecologically selective coloured traps. Ecological Entomology, Cambridge, v. 9, p. 35-4, 1986.

LIESE, W. Preservation of bamboo structures. Ghana Journal of Forestry, Kumasi, v. 15, p. 40-48, 2004.

LOPEZ, O. H. Bambu - su cutivo y aplicaciones en: fabricación de papel, construcción, Arquitetura, Ingeniería, Artesanic. Colômbia: Estudos Técnicos Colombianos Ltda, 1974. p. 41

MARANHÃO, Z. M. Entomologia Geral. São Paulo-SP: Nobel, 1976. p. 96.

NAIR, K. S. S.; MATHEW, G. Dried tapioca tuber for laboratory rearing of the bamboo borer, Dinoderus minutus Fabr. (Coleoptera: Bostrichidae). Journal Material und Organismen. Berlin, v. 19, n. 1, p. 49-54, 1984.

NIEMEYER, H. Field response of Ips typographus L. (Coleoptera: Scolytidae) to different trap structures and white versus black flight barriers. Zeitschrift für Angewandte Entomologie. Hamburg, v. 99, n. 1, p. 44- 51, 1985.

NORHISHAM, A. R.; ABOOD, F.; RITA, M.; HAKEEM, K. $\mathrm{R}$. Effect of humidity on egg hatchability and reproductive biology of the bamboo borer (Dinoderus minutus Fabricius). Springer Plus, v. 2, n. 9, p. 1-6, 2013.

NORDLUND, D. A.; LEWIS, W. J. Terminology of chemical releasing stimuli in intraspecific and interspecific interaction. Journal of Chemical Ecology. Florida, v. 2, p. 211, 1976.

OLIVEIRA, C. R. F.; FARONI, L. R. D.; GUEDES, R. N. C.; PALLINI, A.; GONÇALVES, J. R. Parasitismo de Acarophenax lacunatus (Cross e Krantz) (Prostigmata: Acarophenacidae) sobre Dinoderus minutus (Fabricius) (Coleoptera: Bostrichidae). Neotropical Entomology, Londrina-PR, v. 31, n. 2, p. 245-248, 2002.
OLIVEIRA, L. C. S.; CANEDO, M. D.; MILANO, S. Agentes destruidores da madeira. In: LEPAGE, E. S. Manual de preservação de madeiras. São Paulo-SP: IPT, 1986. p. 99279.

OTT, A. P.; FERRARI, A.; ELY, I.; BINS NETO, R. C.; AZEVEDO FILHO, W. S.; CARVALHO, G. S. Monitoramento de cigarrinhas com armadilha adesiva amarela em pomares de laranja valência nos vales do Cai e Taquari, Rio Grande do Sul, Brasil. Revista Brasileira de Agroecologia. Pelotas-RS, v. 1, n. 1, p.1157-1160, 2006.

PERES FILHO, O.; BARBOSA, J. L.; SOUZA, M. D.; DORVAL, A. Altura de voo de bostriquídeos (Coleoptera: Bostrichidae) coletados em Floresta Tropical Semidecídua, Mato Grosso, Pesquisa Floresta Brasileira, Colombo-RS, v. 32, n. 69, p. 101-107, 2012.

PLANK, H. K.; Biology of the bamboo powder-post beetle in Puerto Rico. Porto Rico: Agricultural Experiment Station, 1948. 29 p. (Boletim Técnico, 44)

PLANK, H. K.; HANGEMAN R. H. Starch and other carbohydrates in relation to powder-post beetle harvested bamboo. Journal of Economic Entomology, Annapolis, v. 44, n. 1, p. 73-75, 1951.

REBOITA, M. S.; GAN, M. A.; ROCHA, R. P.; AMBRIZZI, T. Regimes de precipitação na América do Sul: uma revisão bibliográfica. Revista Brasileira de Meteorologia, São José do Campos-SP, v. 25, n. 2, p. 185-204, 2010.

SINGH, P.; BHANDARI, R. S. Insect pest of bamboos and their control. Bamboos Current Research, Indian, v. 10, n. 14, p. 190-194., 1988.

SPILMAN, T. J. False Powderpost Beetles of the Genus Dinoderus in North América (Coleoptera, Bostrichidae). The Coleopterists Bulletin. Comut, v. 36, n. 2, p. 193-196, 1982.

WILLIANS, C. B.; SINGH, B. P. Effect of moonlight on insect activity. Nature, London, v. 167, n. 4256, p. 853-854, 1951. 